\title{
Smart Device Control Using Augmented Reality
}

\author{
Professor R.K.Moje ${ }^{1}$, Mr Chandan Kumar Shukla ${ }^{2}$, Miss Renuka D Kolhe ${ }^{3}$, Miss Sukriti Bhat ${ }^{4}$ \\ Department of Electronics, College Of Engineering, Manjari $(\mathrm{Bk})^{1,2,3,4}$
}

\begin{abstract}
In recent years, augmented reality technology is being widely used in various areas. Augmented Reality (AR) is basically a real view modified by a computer. It uses computer generated imagery and sounds to enhance one's current perception of reality. While virtual reality describes an imaginary world that exists in computers and our minds, augmented reality is a mixture of real world and virtual world. It is therefore different for the human mind to tell the difference between real world and augmented reality. In this paper, we describe the development of a prototype that uses augmented reality to control electrical devices like fan, motors, AC etc by hand gestures. Up to 8 electrical devices can be controlled. A virtual plane is present between user and display. The user interacts with this virtual plane and hands and fingers are tracked and gestures recognized. These gestures are then used to select the button on the display which are used for selecting a particular device and to turn it on or off as per the requirement of user. This project is very helpful for people of physical limitations like old age people, physically disabled people and also used for industrial and office work.
\end{abstract}

Keywords: Augmented Reality (AR); Virtual Reality (VR); Hand Gesture; Air Touch; Human Computer Interaction (HCI)

\section{INTRODUCTION}

AR creates a live direct or indirect view of a real world in This paper is proposed to utilize the smart Augmented which elements are augmented by computer generated Reality technique in controlling electrical devices that will sensory input [4]. It provides relevant extra information be beneficial for industrial and home applications. The rest about what you are looking at and makes the information of the paper is organised as follows. Section 2 describes about the user interactive and digitally manipulable. Also the proposed system in detail. Section 3 describes system known as Air Touch, this method removes any need to flow and algorithms associated with hand gesture physically touch the display and makes use of computer recognition. Conclusion and future scope are given in vision techniques. Air Touch's driving innovation is to section 4 create a virtual interaction surface in the space, i.e., the "air," between the user and the computer. The user then "touches" this virtual interaction surface, i.e., this air, and his or her actions are mapped in a natural way to the computer system [3]. Many of the gesture-based humancomputer interaction (HCI) [5] systems drawbacks have been overcome by Air Touch as discussed below.

Early approaches to the hand gesture recognition problem involved the use of markers on finger tips. An associated algorithm was used to detect the presence and color of the markers, through which one can identify which fingers are active in the gestures. Also the inconvenience of placing markers on the user's hand made this an infeasible approach in practice. Furthermore, it was mandatory to have different background color with respect to the color of marker because then only it would be possible for the camera to detect the marker points and thus estimate the hand gestures. The other drawback was the limited number of hand gestures .If any gesture apart from fixed number of gesture was performed, then it becomes difficult for the camera to identify that gesture.[2,6,7]

These problems were solved by augmented reality concept .Even if there are same colors in the background and foreground; it hardly matters [8]. Hand gestures are not limited in number. In this user is able to leave and re-enter the camera frame with no effect on their ability to utilize the system. If the user moves their hand out of the view of the camera or stands up for a moment, it has no negative effect.

\section{PROPOSED SYSTEM}

We proposed a system to use augmented reality method in which we assume virtual interfacing plane in between actual hardware and human interface [9]. This environment can be effectively implemented using standard hardware webcam and frame extraction techniques. Figure 1 shows the block diagram of our system. The webcam is interfaced with the PC. It registers the background image as well as scans the user movements i.e. hand gestures.

A region of maximum difference is obtained by subtracting these two images. In that particular region whichever button is pointed out by the hand gestures will be selected and controlling of device will take place. These hand gestures are send as commands by PC to control devices. As shown in the block diagram, microcontroller is used to communicate with PC and control devices. For this purpose, serial communication and interfacing of microcontroller with PC is necessary and is done by RS232 standard and MAX232 IC.

The device drivers are used to provide interfacing between the logic circuitry and peripheral power loads. Hence devices such as fan, ac, motors etc can be controlled in our system. User can select between next device and previous device and accordingly turn them on or off as per the movement of hand gestures which helps to select the buttons on display and perform the action likewise. 


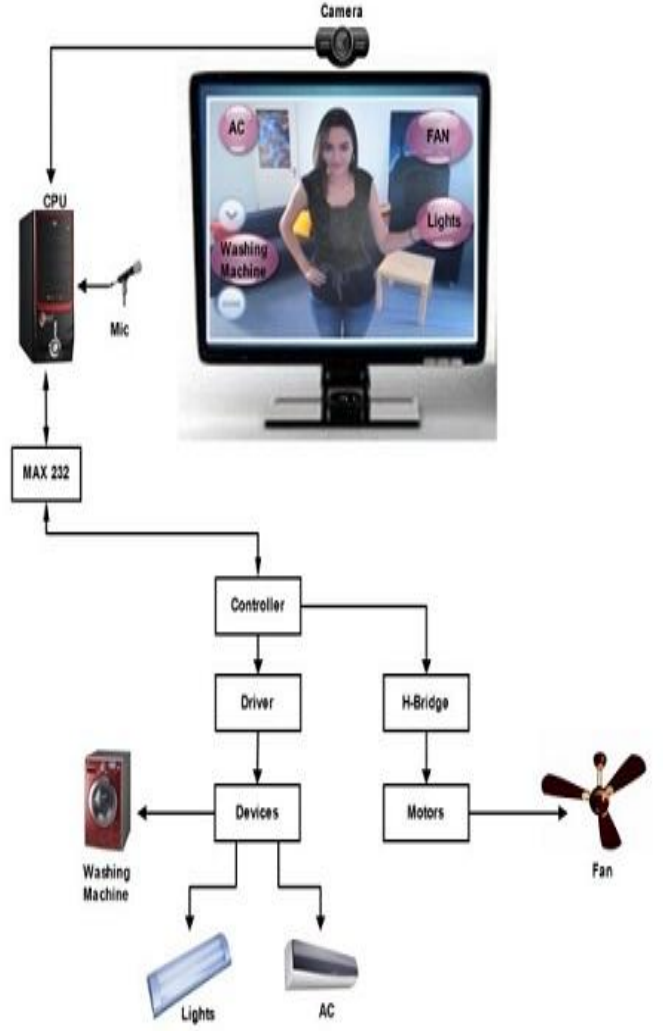

Figure 1. Block Diagram

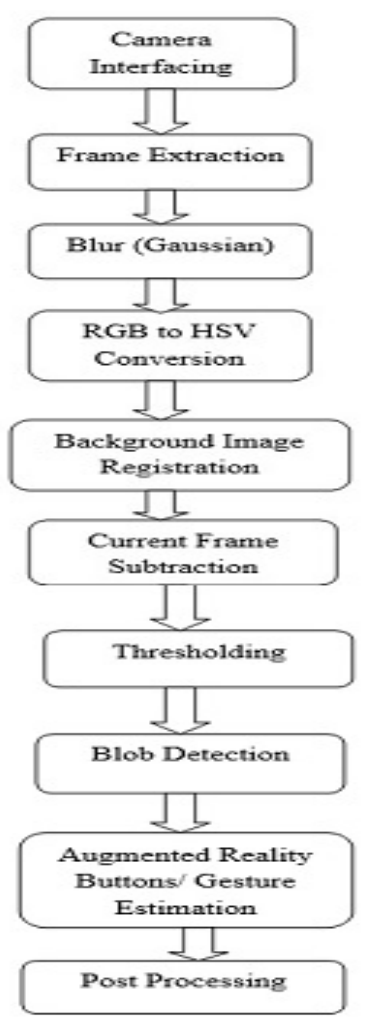

Figure 2. System Flow
III. SYSTEM FLOW

Figure 2 shows system flow of our proposed system. At first, the hand gesture movement is captured by Webcam. Then video frames are captured and send for processing. In processing all captured images are blurred for better detection and from these images all are converted into HSV color model for obtaining accurate color.

The webcam registers the background image and scans user image.

It then subtracts images to get difference between background and current image to get region where there is maximum difference. Next, thresholding for converting image into binary form (black and white image) and the blob detection from images are carried out. From these blobs, gesture is recognized.

The gesture obtained in the region is then used to check for button index and action is performed likewise.

The last step is preprocessing the recognized gestures and according to that gesture command associated with that is send to system and particular device is selected and is then turned on or off.

In order to recognize virtual plane and its function, extraction and approximation algorithms are used, in which blurring, thresholding, RGB to HSV conversion and blob detection etc. principles are used.

Finally we will analyze, detect and recognize the hand gesture.
Now we will discuss Hand gesture detection and recognition in brief one by one.

\section{A. Blur an Image:}

\section{ALGORITHM}

In image terms blurring means that each pixel in the source image gets spread over and mixed into surrounding pixels. Another way to look at this is that each pixel in the destination image is made up out of a mixture of surrounding pixels from the source image. Blurring an image reduces the sharpening effect; this makes the detection more accurate. We split all RGB value separately and Calculate the RGB average of surrounding pixels and assign this average value to it. Repeat this above step for each pixel and finally we get blurred images of Hand Gestures. We are using Gaussian blur in our project. A Gaussian blur (also known as Gaussian smoothing) is the result of blurring an image by a Gaussian function. It is a widely used effect in graphics software, typically to reduce image noise and reduce detail. Gaussian smoothing is also used as a pre-processing stage in computer vision algorithms in order to enhance image structures at different scales.

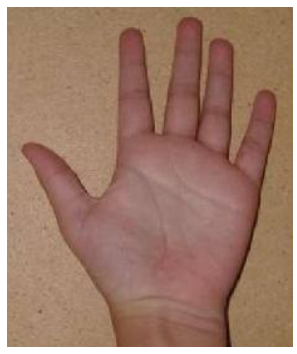

(a) Original Image

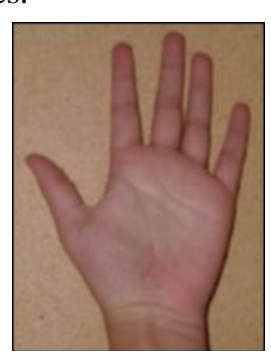

(b) Blur Image
Figure 3.Blur an Image 
B.RGB TO HSV (Grayscale) Conversion:

After blurring all images, all blurred images are transferred into HSV (Hue, Saturation, Value) model. HSV is stronger model than RGB because it offers a more intuitive representation of the relationship between colors. HSV selects more specific color. In HSV model value of " $\mathrm{H}$ " and "S" remain constant if the value of " $\mathrm{V}$ " changes, but value of RGB changes with the change in , $\mathrm{V}^{\text {ee }}$. So we get True color value. Figure 4(a) shows input image, 4(b) shows image with Hue, 4(c) saturated image,4(d) represent Value through which we get grayscale image.

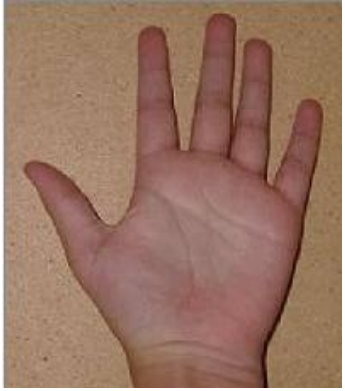

(a) Input Image

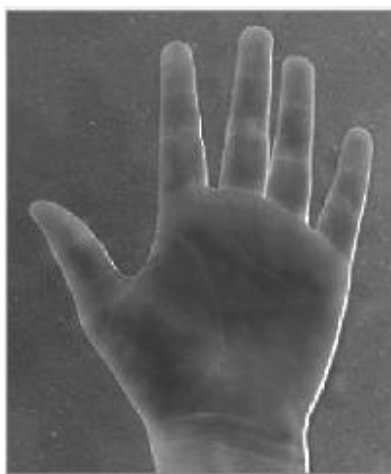

(c) Saturation

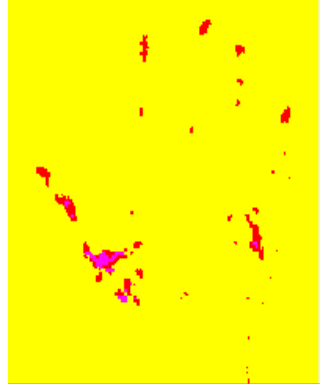

(b) Hue

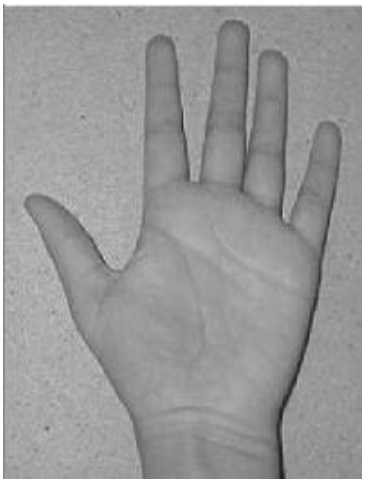

(d) Value
Figure 4. RGB to HSV Conversion

\section{Image Thresholding.}

For recognizing Hand Gesture we use Thresholding. Image segmentation can be easily done by the simplest method i.e. thresholding. From a grayscale image, we use thresholding method that can be used to create binary images i.e. image which have only 2 colors, black or white. It is usually used for gesture extraction where required gesture from an image are converted to white and everything else to black (or vice-versa).

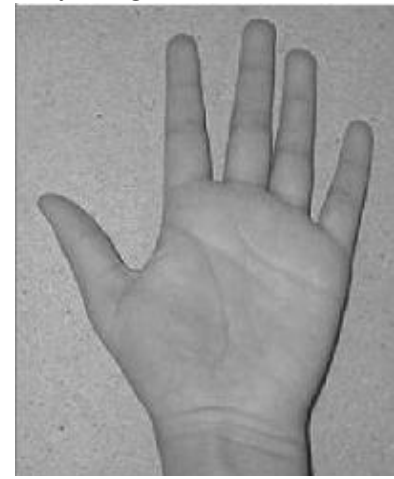

(a) Input Image

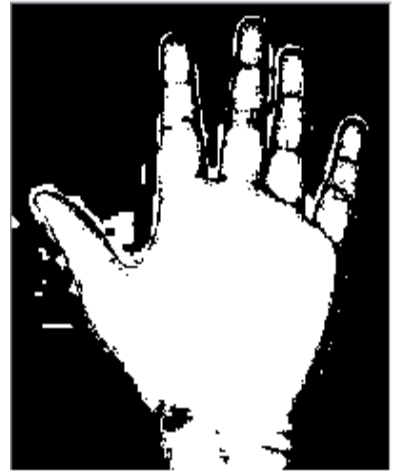

(b) Threshold Image
Figure 5. Image Thresholding

\section{Blob detection}

After getting binary image in the form of only Black and White, we get white blob and rest black (Vice versa), we must have to detect these blobs. For detecting blob firstly, Starts from the first line of the image and find groups of one or more white (or black) pixels. Group of one or more white pixels are called as line blobs. Find X, Y coordinates of each those blob. Number each of these groups. Repeat this sequence on next line. While you are collecting the line blobs, check whether the line blobs that checked before this current line and see if these blobs overlap each other. If so, you merge these line blobs by using their $\mathrm{X}$ and $\mathrm{Y}$ co-ordinates to one blob it will be treated as a whole blob. Repeat this for every line and you have a collection of blobs.

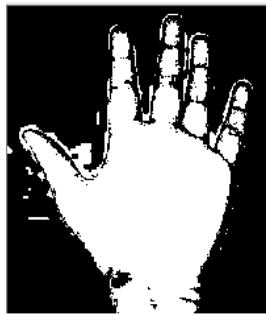

Figure 6. Blob Detection

\section{E. Gesture Recognition}

The gesture-recognition module tracks the moving hand features by using Image Subtraction, identifies the motion, and determine which device is selected. Then the recognized gesture is then send to the application being used which is detect which button is chosen and what response is associated to that button, which then communicates with the Augmented Reality.

\section{RESULT}

This is the result window of our project .From the window, wecan control upto 8 electrical devices.

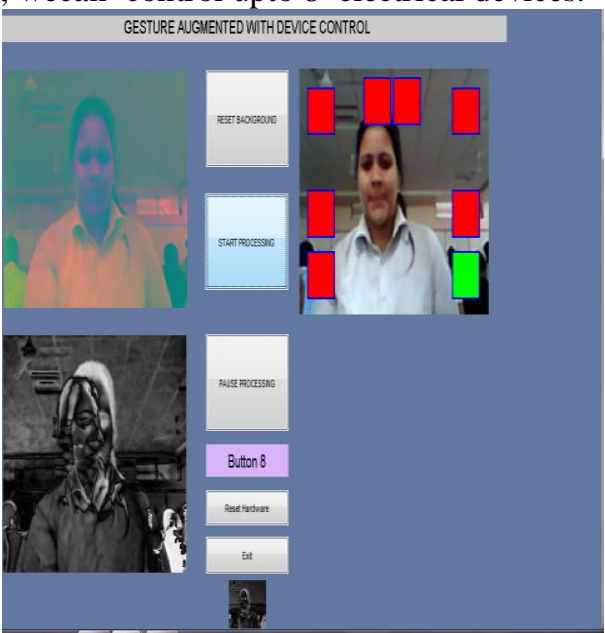

Figure 7 Result window

VII. CONCLUSION AND FUTURE WORK

This paper implements a system for smart use of augmented reality in controlling electrical devices. This system is robust, easy to use and enables the user to control the devices without being in physical contact with them. This system is highly beneficial for old age and 
physically disabled people. It is also useful for industrial applications. As future work, apart from hand gestures to control the device, voice can also be added with the help of microphones for controlling devices. This can be especially beneficial for the people who does not have hands. Also 3D camera can be used in the future system for better resolution of image and also with the use of these cameras the users hand gestures in front of the camera and away from it are considered as separate images. Because of this, accuracy of the system is increased.

\section{REFERENCES}

[1] Ahmed Mohmmad Ullah, Md. Rashedul Islam,Remote Touch: "Augmented Reality Based Marker Tracking For smart Home Control",2013

[2] Ching-Hao Lai, "A Fast Gesture Recognition Scheme for RealTime Human Machine Interaction System",in Conference on Technologies and Applications of Artificial Intelligence, pp. 212217,2011

[3] Daniel R. Schlegel, Albert Y. C. Chen, Caiming Xiong,Jeffery A.Delmerico, "Air Touch: Interacting With Computer Systems At A distance", pp 1-8,2010.

[4] Yoki Ariyana, Aciek Ida Wuryandari, "Basic 3D Interaction Techniques in Augmented Reality" in International Conference on System Engineering and Technology, September 11-12, 2012, Bandung, Indonesi,2012.

[5] Jinhua Zeng, Yaoru Sun and Fang Wang, "A natural hand gesture system for intelligent human-computer interaction and medical assistance", in Third Global Congress on Intelligent Systems, pp.382-385,2012

[6] Ankita Saxena,Deepak Kumar Jain,Ananya Singhal "Hand Gesture Recognition using an Android Device", In Fourth International Conference on Communication Systems and Network Technologies, pp.819-822

[7] Yikai Fang, Kongqiao Wang, Jian Cheng and Hanqing Lu, "A Real-Time Hand Gesture Recognition Method", in Proceedings of the IEEE International Conference on Multimedia and Expo, pp. 995-998, 2007.

[8] Jens Lambrecht1 and Jorg Kruger "Spatial Programming for Industrial Robots based on Gestures and Augmented Reality",2012. 